\title{
On the quantum theory of isotope effects in the electromigration and thermomigration of light interstitials
}

\author{
A M Stoneham and C P Flynn $†$ \\ Theoretical Physics Division. AERE, Harwell, Berkshire, UK
}

MS received 26 September 1972

\begin{abstract}
The electromigration and thermomigration of light interstitials, such as hydrogen in metals, have been examined in terms of the quantum theory of diffusion of Flynn and Stoneham. The pronounced isotope effect observed in the effective charge $Z^{*}$ is shown to come from the differences in the self trapping distortions of the various isotopes. The qualitative dependence of $Z^{*}$ on isotope, host lattice and temperature are correctly predicted. The interstitial contribution to the reduced heat of transport $Q^{*}$ is calculated directly from the quantum theory. There is no term proportional to the activation free energy in the régime of interest. The dominant contribution to $Q^{*}$ is probably that from the electron gas, although the entropy of solution adds an important term. Again, the qualitative results are consistent with the sparse experimental data.
\end{abstract}

\section{Introduction}

The diffusion rates of light interstitials, notably hydrogen, carbon, nitrogen and oxygen, have been measured in a number of metallic host lattices (for a review, see Wert 1970). For hydrogen, the striking isotope dependences observed in the interstitial jump rate are widely attributed to quantum effects. Recent results are summarized in the proceedings of the 1972 Jülich conference 'Hydrogen in Metals'.

The present paper is concerned with the isotope effects in the heat and charge fluxes accompanying interstitial motion. Reviews of electromigration and thermomigration are given by Howard and Lidiard (1964), Lidiard (1966), Allnatt and Chadwick (1967), Huntington (1969) and Oriani (1969); other articles will be found in the proceedings of the 1970 Marstrand meeting. An isotope dependence of the heat flux has been observed by Gonzalez and Oriani (1965) for $\mathrm{H}$ and $\mathrm{D}$ in Fe and Ni, and by Wipf (1972) for $\mathrm{H}$ and $\mathrm{D}$ in $\mathrm{Nb}$. The isotope effect in charge flux has been detected by Oriani and Gonzalez (1967) for $\mathrm{H}$ and $\mathrm{D}$ in $\mathrm{Fe}$ and $\mathrm{Ni}$.

It is observed in the several reviews that both the heat and charge fluxes which accompany the interstitial motion contain major contributions from the effect of atomic migration on the host electron gas. Since the coupling to the electron gas depends on details of the defect-electron interaction that cannot be analysed with sufficient accuracy at present, it is not yet possible to calculate the magnitudes (nor often the sign) of the heat and charge fluxes. Instead we concentrate on the experimentally observed isotope dependence and the qualitative way in which these established effects can arise. It should

\footnotetext{
† Permanent address: Physics Department, University of Illinois, Urbana, Illinois 61801, USA.
} 
be emphasized at the outset that no isotope effects arise in classical theory. Our interpretation is based on the quantum theory of interstitial hopping developed by Flynn and Stoneham (1970) and on related work for the small polaron problem. These methods have been successful in explaining many aspects of the observed behaviour of light interstitials.

\subsection{Electromigration}

In an isotropic solid, the interstitial charge flux $J_{\mathrm{q}}$ is specified by an 'effective charge' $Z^{*}$ defined by

$$
Z^{*}|e|=J_{\mathrm{q}} / J_{\mathrm{p}}
$$

where $J_{\mathrm{p}}$ is the particle flux. $Z^{*}$ differs from the ionic charge (unity for hydrogen) by contributions arising from electronic motion. Its magnitude is discussed by Gerl (1971) and Fiks (1971).

The isotope effect in $Z^{*}$ finds no satisfactory explanation in classical theory. Isotopes are chemically similar, so that the potential energy of the crystal as a function of configuration does not depend on isotopic mass. In the rate theory formulation of diffusion employing classical statistical mechanics, the probabilities with which configurations occur are also independent of isotopic mass. The isotope effect in $Z^{*}$ for light interstitials vanishes whenever the particle velocity is sufficiently small compared with the velocities of the electrons which it scatters. Consequently Oriani and Gonzalez suggested a breakdown of the Born-Oppenheimer approximation to explain the observed isotope effect in terms of ionic recoil. This seems unlikely, partly because the proton mass is so much larger than that of the electron, and partly because one would expect an isotope effect in the residual resistivity which is not observed. In $\S 2$ we attribute the isotope effect to the differences in jump configurations for the different isotopes.

\subsection{Thermomigration}

The heat of transport $Q$ defines the heat flux $J_{\mathrm{h}}$ accompanying a particle flux by the relation

$$
Q=J_{\mathrm{h}} / J_{\mathrm{p}}
$$

but this includes the enthalpy of solution of the moving particles. The actual driving force in a temperature gradient contains instead

$$
Q^{*}=Q-H
$$

with $H$ the enthalpy of solution. The reduced heat of transport $Q^{*}$, contains three major contributions. The first comes from the strong (nonclassical) isotope dependence of the entropy of solution. The second arises because the electron flow accompanying the interstitial flux does not carry a thermal average distribution of electrons. This "electron wind' term may be isotope dependent for the same reasons as $Z^{*}$. Finally, there is a term associated with the interstitial itself. This is calculated from first principles in $\S 3$.

\subsection{Quantum theory of diffusion}

We shall use the quantum theory of the diffusion of light interstitials proposed by Flynn and Stoneham. Stoneham (1972) has recently reviewed the theory and its relation to 
experiment. The important features are the self trapping distortion and its consequences, and the qualitative differences between the cases of bcc and fcc host lattices.

The theory contains these basic approximations:

(i) The Born-Oppenheimer approximation is used to ensure that there is a unique potential for each configuration of the system;

(ii) It is assumed that the interstitial is so much lighter than the host atoms that it can follow their motion adiabatically. The interstitial gives rise to a force on the lattice which varies smoothly with lattice configuration;

(iii) The interstitial energy is taken to vary linearly with displacements of the host lattice:

(iv) The transitions between sites are induced by interstitial matrix elements $J$ independent of the host lattice coordinates; there are no matrix elements of the interstitial-lattice coupling that connect interstitial states in different interstices. $J$ is essentially the matrix element of interstitial kinetic energy between states on adjacent sites.

When an interstitial is introduced into a particular interstice the defect-lattice coupling causes a 'self trapping' distortion. The free energy change is

$$
F_{\mathrm{t}}=-\frac{1}{2} \sum_{q} M \omega_{q}^{2}\left|\boldsymbol{x}_{q}^{0}\right|^{2}
$$

where $M$ is the host atom mass, $\omega_{q}$ the frequency of host lattice mode $q$ and $\boldsymbol{x}_{q}^{0}$ gives the self trapping displacements. The self trapping distortion has a profound effect on the defect motion. If there were no distortion the proton would propagate through the rigid lattice, as do electrons in normally conducting crystals. The band width for this motion would be $2 n J$, where $n$ is the coordination number of the interstitial sites. This propagating picture holds when $|2 n J| \gg\left|F_{\mathrm{t}}\right|$. In the model of Flynn and Stoneham, $\left|F_{\mathrm{t}}\right| \gg|2 n J|$, and in this régime the interstitial hops from site to site.

When host lattice atoms straddle the jump path, the transition matrix element cannot be considered independent of their position. This is true for the fcc hosts, where the assumption (iv) must be generalized (Flynn and Stoneham 1970). In consequence activation energies for hydrogen in fcc hosts $(0.25-0.45 \mathrm{eV})$ are systematically higher than those for bcc hosts $(0.05-0.20 \mathrm{eV})$ where the self trapping distortion primarily determines the hopping rate.

\section{Electromigration}

As mentioned above, an isotope dependence of $Z^{*}$ is observed, although there appears no reason to expect one within classical diffusion theory. It is interesting to observe that such an isotope effect appears naturally within the quantum theory of diffusion. The important features are first that the self trapping distortion will be different for different isotopes, and second that the 'electron wind' term from electron scatter depends on the detailed configuration of the interstitial and the neighbouring host atoms.

To see how self trapping affects $Z^{*}$, it is useful to contrast electromigration with ionic conductivity in insulators. A charge $Z|e|$ migrating through $l$ between adjacent sites in an insulator absorbs energy $Z|e| \boldsymbol{I} \cdot \boldsymbol{E}$ from an external field $\boldsymbol{E}$. The modified jump rates, $W_{+}$with and $W_{-}$against the field, may be calculated from the specific formulae of Flynn and Stoneham or by use of the principle of detailed balance. The result: 


$$
W_{ \pm}=W_{0}(1 \mp Z|e| \boldsymbol{l} \cdot \boldsymbol{E} / k T)
$$

with $W_{0}$ the unperturbed rate, depends only on the initial and final sites. However, in electromigration in metals, the direct effect of $\boldsymbol{E}$ is augmented by an 'electron wind' force which depends on the specific configuration through which the system is passing, since the electron scattering depends on the interstitial and lattice coordinates. In contrast to the case for insulators, the work done by external fields in metallic hosts depends on the particular path taken by the system during the jump. For a light interstitial, the lattice coordinates $\boldsymbol{x}$ remain almost constant during the fast interstitial jumps. We may therefore identify the particular value of the effective charge as a function $Z^{*}(x)$ of these coordinates alone. The observed $Z^{*}$ is then a thermal average of $Z^{*}(x)$ over accessible jump paths. This averaging may lead to a temperature dependence of $Z^{*}$ and of deviations from Matthiesen's rule.

Although $Z^{*}(x)$ cannot be calculated from first principles yet, some of its properties can be deduced from symmetry arguments. The lattice displacements may be placed in two classes. Members $\boldsymbol{x}_{\mathrm{a}}$ of one class are antisymmetric about the midpoint of the jump path, and the remainder $\boldsymbol{x}_{\mathrm{s}}$ are symmetric (for boc lattices the interstitial site is not cubic and its axis is rotated by the jump; this complicates the description without introducing problems of principle). Now $Z^{*}(\boldsymbol{x})$ must be even in $\boldsymbol{x}_{\mathbf{a}}$, for the jump $\boldsymbol{l}$ in a field $\boldsymbol{E}$ for configuration $\boldsymbol{x}_{\mathrm{a}}$ is exactly equivalent to the jump $-\boldsymbol{l}$ in a field $-\boldsymbol{E}$ for configuration $-\boldsymbol{x}_{\mathrm{a}}$, and separate reversals of $\boldsymbol{E}$ and $\boldsymbol{l}$ must reverse the sign of the work $\epsilon$ done by the electron gas:

$$
\begin{aligned}
\epsilon\left(\boldsymbol{x}_{\mathrm{a}}, l, E\right) & =\epsilon\left(-\boldsymbol{x}_{\mathrm{a}},-\boldsymbol{l},-E\right) \\
& =-\epsilon\left(-\boldsymbol{x}_{\mathrm{a}}, l,-E\right) \\
& =\epsilon\left(-\boldsymbol{x}_{\mathrm{a}}, l, E\right) .
\end{aligned}
$$

Thus $\epsilon$ is even in $\boldsymbol{x}_{\mathrm{a}}$, and so also is $Z^{*}\left(\boldsymbol{x}_{\mathrm{a}}\right)$. In contrast, the work $\epsilon$ is neither even nor odd in $\boldsymbol{x}_{\mathrm{s}}$.

An important point concerning the isotope dependence of $Z^{*}$ now emerges from the different roles of $x_{\mathrm{a}}$ and $\boldsymbol{x}_{\mathrm{s}}$ in the jump process. The mean values of the $\boldsymbol{x}_{\mathrm{a}}$ are reversed by the jump, and these modes enter into the jump rate through lattice overlap integrals dominated by $\boldsymbol{x}_{\mathrm{a}} \simeq 0$. Since $Z^{*}(\boldsymbol{x})$ contains no terms linear in $\boldsymbol{x}_{\mathrm{a}}$, isotopic differences among the $x_{\mathrm{a}}$ are unlikely to cause any substantial isotope dependence of $Z^{*}$. These isotope effects must arise from symmetric displacements $x_{\mathrm{s}}$, since $Z^{*}(x)$ contains terms linear in $\boldsymbol{x}_{\mathrm{s}}$ and the transitions are not dominated by $\boldsymbol{x}_{\mathrm{s}} \simeq 0$. Jumps in boc lattices are likely to occur close to the symmetric displacements $\boldsymbol{x}_{\mathrm{s}}^{0}$ associated with the self trapping distortion of a particular isotope. In fcc lattices the important values of $\boldsymbol{x}_{\mathrm{s}}$ will be determined partly by the self trapping distortions and partly by the dependence of the transition matrix element on the symmetric motion of the atoms straddling the jump path. Isotope differences in $Z^{*}$ are thus identified with the isotope dependence of the symmetric coordinates and the consequent changes in electron scattering.

Certain qualitative points can be made, even though cross sections are not available at present for quantitative analysis. The self trapping distortion increases with zero point energy and with decreasing isotopic mass. (Flynn and Stoneham 1970, Matthew 1970). The resistivity and the electron drag, measured by $\left(1-Z^{*}\right)$ for hydrogen, are likely to increase with lattice distortion. Thus $\left(1-Z^{*}\right)$ is expected to be larger for protons than deutrons. Observed values support this view 


$$
\begin{aligned}
\left(1-Z^{*}\right)_{\mathrm{H}} /\left(1-Z^{*}\right)_{\mathrm{D}} & =1.24(\mathrm{bcc} \mathrm{Fe}) \\
& =1.625(\text { fcc Ni, 475 K) } \\
& =1.50(\text { fcc Ni, 625 K). }
\end{aligned}
$$

Further, the greater importance of symmetric distortions in fce hosts through their effect on the transition matrix element may tend to give a larger and more temperature dependent isotope effect than in bcc hosts. These features are in agreement with available results for bcc $\mathrm{Fe}$ and $\mathrm{fcc} \mathrm{Ni}$, but more data are required before firm conclusions can be drawn.

\section{Thermomigration}

If the isotope dependence of $Q^{*}$ arose entirely from the electron scatter terms affecting $Z^{*}$, then $Q^{*} /\left(1-Z^{*}\right)$ should be independent of isotope. This is not the case, so we must examine contributions to $Q^{*}$ from the interstitial and its coupling to the lattice. We shall show that, in the hopping regime studied by Flynn and Stoneham, there are no terms in $Q^{*}$ associated with the hopping energy, and that only the entropy of solution contributes to the remaining isotope effect in $Q^{*}$. This is not the case in other regimes of diffusion and is contrary to common assertions in classical and phenomenological theories.

The calculation is closely related to that of Sumi (1971) of the absolute thermopower of small polarons, to which the reader is referred for technical details. After some simple generalizations, such as allowing linear coupling to all lattice modes to contribute to the self trapping distortion, his model becomes that of $\S 1.3$. Here we give only the main physical points and the results.

Sumi's calculation of the thermopower uses the Kubo formalism (eg Sumi 1971, Appel 1968) in which the expectation value of the current is determined to lowest order in the driving perturbation. For interstitial diffusion this is the matrix element $J$, related earlier to the bandwidth in a rigid host; there are no lattice matrix elements driving diffusion in this mode. It is assumed that $J$ is independent of the antisymmetric displacements $\boldsymbol{x}_{\mathrm{a}}$. We have generalized the calculation to allow $J$ to depend on the symmetric displacements $\boldsymbol{x}_{\mathrm{s}}$, as appears necessary for fcc hosts. By selecting appropriate expressions for the heat and particle currents, one may extract the following results for the diffusion parameters.

\subsection{Hopping régime}

This is the regime of strong localization. discussed by Flynn and Stoneham. Here the self trapping free energy $F_{\mathrm{t}}$ is much larger than the rigid-lattice bandwidth $2 n \mathrm{~J}$. At high temperatures the diffusion rate varies as

$$
J^{2} T^{-1 / 2} \exp \left(-F_{\mathrm{a}} / k T\right)
$$

where the activation free energy:

$$
F_{\mathrm{a}}=\frac{1}{2} \sum_{q} M \omega_{q}^{2}\left|\frac{1}{2} \Delta x\right|^{2}
$$

involves the change $\Delta x$ in the equilibrium lattice coordinates. $F_{\mathrm{a}}$ clearly depends only on the antisymmetric parts of the self trapping displacements. The heat of transport $Q$ 
proves to be simply given by $F_{t}$, the value of the excess free energy of the interstitial. Consequently the reduced heat of transport is just

$$
Q_{\AA}^{*}=-T S
$$

with $S$ the partial excess entropy of solution. There is no term in the activation free energy $F_{\mathrm{a}}$. This is still true when the matrix element $J$ is allowed to depend on the symmetric displacements $\boldsymbol{x}_{\mathrm{s}}$. Any systematic trends in $Q_{\mathrm{A}}^{*}$ between bcc and fcc hosts must come from $S$ and not from the differences in $F_{\mathrm{a}}$ mentioned in $\S 1.3$. The fact that the thermopower similarly contains no terms in the activation energy has been widely acknowledged; this arises from the fact that the interstitial properties contain no significant contributions from the band like states (Sewell 1963, Schotte 1967, Efros 1967, Klinger 1968, Austin and Mott 1969 p 61).

\subsection{Propagating régime}

In this régime, self trapping is not dominant. The interstitials propagate through the lattice, rather than diffusing by a hopping motion. The reduced heat of transport is found to be

$$
Q_{\mathrm{B}}^{*}=2 k T
$$

similar to the result for an ideal gas. It appears because most transport takes place through the faster moving carriers of thermal energy. This régime is unlikely to be of any concern for the interstitial systems considered here.

\subsection{Intermediate régime}

Over a restricted range of parameters the interstitials are localized by self-trapping, but transport occurs through excited band states rather than by tunnelling as in $\S 3.1$. Diffusion is thermally activated with an energy

$$
F_{\mathrm{C}}=\left|F_{\mathrm{z}}\right|-|n: J| \text {. }
$$

The reduced heat of transport obtained is

$$
Q_{\mathrm{C}}^{*}=F_{c}+2 k T \text {. }
$$

It contains both a kinetic contribution and a term involving the activation energy. The intermediate regime corresponds to Sussmann's (1971) model of diffusion.

We can make only a qualitative comparison of the present results with experiment, for our understanding of the important electronic contribution is incomplete. Only the hopping and intermediate régimes appear plausible for accessible temperature ranges, so we shall ignore the propagating régime. One piece of evidence points to the hopping régime for diffusion rather than the intermediate régime. Wipf has found a slope change in the logarithm of the diffusion coefficient as a function of inverse temperature for $\mathrm{Nb}: \mathrm{H}$, but finds no comparable anomaly in $Q^{*}$. This is inconsistent with equations (3.5) and (3.6), and suggests a hopping process of the type discussed in $\$ 3.1$. The intermediate régime is, in any case, only relevant over a restricted range of physical parameters. The remaining discussion assumes that the régime of strong localization discussed by Flynn and Stoneham does, in fact, prevail. 
The dominance in $Q^{*}$ of the electron scattering contribution is suggested by the fact that $Q^{*}$ has the same sign for various interstitials in the same host. Examples are:

$\begin{array}{lll}\alpha-\mathrm{Fe} \text { (bcc) } & \text { H, D, C, N } & Q^{*}<0 \\ \beta-\mathrm{Zr} \text { (bcc) } & \text { H, N, O } & Q^{*}>0 \\ \mathrm{Nb} \mathrm{(bcc)} & \text { H, D } & Q^{*}>0 \\ \mathrm{Ni} \mathrm{(fcc)} & \text { H, D } & Q^{*}<0 .\end{array}$

The results are consistent with the view that one sign of charge carrier provides the major driving force for all interstitials in a particular host. Moreover, the observed values of $Z^{*}$ are uniformly positive (with the possible exception of $\gamma-\mathrm{Fe}: \mathrm{N}$ ), so that $Q^{*} / Z^{*}$ has a unique sign for a given host lattice. The temperature dependence $Q^{*} \sim Z^{*} T^{2}$ (eg Gerl 1971) anticipated from simple models of the electron-lattice coupling is not observed. However, it does seem that the main trends of $Q^{*}$ among materials are linked to the properties of the host electron gas.

Nevertheless, the isotope effect in $Q^{*}$ shows clearly that other properties are important. In $\mathrm{Nb}$, for example, $Q_{\mathrm{D}}^{*}$ and $Q_{\mathrm{H}}^{*}$ are both positive and in $\mathrm{Fe}$ both are negative. But in both cases $Q_{\mathrm{D}}^{*}-Q_{\mathrm{H}}^{*}$ is positive. Moreover, there appears to remain a large isotope dependence of $Q^{*} /\left(1-Z^{*}\right)$, which expresses the ratio of two terms proportional to the electron scattering, and which might be presumed insensitive to isotopic differences. There are no obvious trends between bcc and fcc hosts.

Our analysis above suggests these effects may come from the lattice contribution $Q_{\mathrm{A}}^{*} \equiv-T S$ given in (3.3). The magnitude of $S$, the partial excess entropy of solution, is somewhat obscured for hydrogen in metals by questions of site occupancy. An abundance of experimental data (Ebisuzaki and O'Keefe, 1967) indicates that the entropy change on solution is about $-13 \mathrm{cal} \mathrm{mol}^{-1} \mathrm{~K}^{-1}$ in many bcc metals. Of this about $2 \mathrm{calmol}^{-1} \mathrm{~K}^{-1}$ can be attributed to $S$. Thus one obtains a contribution to $Q^{*}$ linear in temperature and of about $0.1 \mathrm{eV}$ magnitude at $1000 \mathrm{~K}$. This is comparable with the observed $Q^{*}$, and the strong temperature dependence is sufficient to invalidate any interpretation of the observed temperature dependence of $Q^{*}$ in terms of the electronic contribution alone.

Unfortunately, existing thermodynamic data do not permit an accurate estimate of the isotope effect in the excess entropy of solution. The entire solubility difference between $H$ and $D$ at $700 \mathrm{~K}$ is typically $\sim 25 \%$ (Ebisuzaki, Kass and O'Keefe 1967 , Eichenauer, Loser and Witte 1965). If this is ascribed entirely to the excess entropy of solution, we obtain $\Delta S \sim 0.3 k$ for the isotope effect, and $\Delta Q^{*} \sim 0.02 \mathrm{eV}$ from (3.3). This is indeed the order of magnitude of the observed isotope effect in $Q^{*}$ for both $\mathrm{Nb}$ and $\mathrm{Ni}$ host lattices. Thus the intrinsic isotope effect in $Q^{*}$ for interstitial migration appears sufficient to alter significantly the contributions from the interactions of the interstitial and the electron gas.

In conclusion, we remark that previous studies of the lattice contribution to thermomigration are based uniformly on classical mechanics and proceed by intuitive simplifications which contradict the present theoretical view of the behaviour of light interstitials. This includes the model of Wirtz (1943) and Brinkman (1954), together with later elaborations by Shewman (1958) and Huntington (1968), and the phenomenological treatments that reduce to similar terms (eg Weeks and Schuler 1972). The WirtzBrinkman model is criticized by Allnatt and Chadwick (1967) and by Oriani (1969). Some discussion of phonon scattering as an added driving force is presented by Schottky 
(1965), Allnatt and Chadwick (1967), Gerl (1971) and Huntington (1968), but the latter author shows this contribution to be small if indeed it exists. In contrast the present treatment of the lattice term is rigorously developed from a well defined model that conforms to the present understanding of non radiative processes. It leads to specific predictions that are certainly valid in the small polaron limit of light interstitial motion and which relate clearly to similar accepted results concerning thermoelectricity in polaron systems.

\section{Summary}

This discussion of isotope effects in electromigration and thermomigration has been restricted by our present inability to deal quantitatively with the important terms arising from the coupling between the electron gas and the moving particle. For electromigration however we have identified the source of isotope effects in $Z^{*}$ within the Born-Oppenheimer approximation in terms of the quantum theory of diffusion of Flynn and Stoneham. In the case of thermomigration we have derived explicit expressions within the same framework for the intrinsic contribution to the reduced heat of transport, $Q^{*}$. There are no terms containing the activation energy within the regime of interest, although substantial and isotope-dependent contributions to $Q^{*}$ arise from the entropy of solution.

\section{References}

Allnatt A R and Chadwick A V 1967 Chem. Rev. 67 681-705

Appel J 1968 Solid State Physics vol 21 eds F Seitz and D Turnbull (New York: Academic Press) pp 193-391 Austin I G and Mott N F 1969 Adv. Phys. $1841-102$

Brinkman J A 1954 Phys. Rev. 93343

Ebisuzaki Y, Kass W J and O'Keefe M $1968 \mathrm{~J}$. Chem. Phys. 49 3329-32

Ebisuzaki Y and O'Keefe M 1967 Prog. Solid St. Chem. 4187

Efros A L 1967 Sov. Phys.-Solid St. $9901-5$

Eichenauer W, Lösser W and Witte H 1965 Z. Metallk. 56287

Fiks V B 1971 Atomic Transport and Diffusion in Solids eds A Lodding and T Lagerwall (Tübingen: Verlag der Z. für. Naturf.) pp 3-8

Flynn C P and Stoneham A M 1970 Phys. Rev. B1 3966-78

Gerl M 1971 Z. Naturf. 269 1-10

Gonzalez O D and Oriani R A 1965 Trans AIME 233 1878-86

Howard R E and Lidiard A B 1964 Rep. Prog. Phys. 27 161-240

Huntingdon H B $1968 \mathrm{~J}$. Phys. Chem. Solids 29 1641-51

___ 1969 Trans. AJME $2452571-9$

Klinger M 11968 Rep. Prog. Phys. 31 225-304

Lidiard A B 1966 Thermoa'ynamics vol 2 (Vienna: IAEA) p 3

Matthew J A D 1970 Phys. Stat. Solidi $42841-7$

Oriani R A 1969 J. Phys. Chem. Solids $30339-51$

Oriani R A and Gonzalez O D 1967 Trans. AIME $2391041-46$

Schotte K D 1967 Z. Phys. 196 393-414

Schottky G 1965 Phys. Stat. Solidi $8357-68$

Sewell G L 1963 Phys. Rev. $129597-603$

Shewman P G 1958 J. Chem. Phys. 29 1032-6

Stoneham A M 1972 Ber. der. Bunsen Ges. fur. Phys. Chem. 76 816-23

Sumi H 1972 J. Phys. Soc. Japan 33 327-342

Sussmann J A 1971 Ann. Phys., Paris 6 135-57

Weeks J D and Schuler K E 1972 J. chem. Phys. 56 1883-9

Wert C A 1970 J. Phys. Chem. Solids 31 1771-84

Wirtz K 1943 Z. Phys. 44221

Wipf H 1972 Hydrogen in metals Jülich Rep. JULConf.-6 II pp 437-48 